\title{
Long-term outcomes of viscocanalostomy and phaco-viscocanalostomy in patients with narrow angle glaucoma
}

\author{
Tasmin Berman $^{1}\left[\right.$ [ $\cdot$ Tobi Somerville $^{1} \cdot$ Anshoo Choudhary $^{1}$
}

Received: 26 August 2021 / Revised: 5 November 2021 / Accepted: 10 November 2021 / Published online: 24 November 2021

(C) The Author(s), under exclusive licence to Springer-Verlag GmbH Germany, part of Springer Nature 2021

\begin{abstract}
Purpose To assess the outcomes of viscocanalostomy and phaco-viscocanalostomy in patients with narrow angle glaucoma at a single tertiary eye centre.

Method All patients undergoing viscocanalostomy for narrow angle glaucoma between June 2010 and June 2017 with a minimum follow-up of 12 months were included. Data was analysed from a prospectively maintained surgical outcome database. Primary outcome was a change in intraocular pressure (IOP). Secondary outcomes were changes in LogMAR visual acuity, number of eye drops, post-operative complications and further surgical interventions. Success was defined at two IOP cut-off points: IOP $\leq 21 \mathrm{mmHg}$ and IOP $\leq 15 \mathrm{mmHg}$ with (qualified success) or without (complete success) drops. Failure was any repeat glaucoma surgery or loss of light perception.

Results Seventy eyes of 46 patients with a mean follow-up of 41.31 months (range 12-60 months) were included. Mean IOP changed from $25.7 \pm 9.6$ to $15.2,15.6,14.6,13.8$ and $14.0 \mathrm{mmHg}$ at $1,2,3,4$ and 5 years post-operatively. Drops reduced from $3.2 \pm 1.1$ pre-operatively to 0.5 at 1 year and 1.1 at all time points thereafter. Qualified success for an IOP $\leq 21 \mathrm{mmHg}$ was achieved in $94.2 \%, 88.1 \%, 92.5 \%, 91.1 \%$ and $92.0 \%$ and complete success in $63.8 \%, 37.3 \%, 30.2 \%, 22.2 \%$ and $24.0 \%$ in years 1 to 5, respectively. Qualified success for an IOP $\leq 15 \mathrm{mmHg}$ was achieved in 53.6\%, 60.9\%, 69.8\%, 68.9\% and 64.0\% and complete success in $39.1 \%, 26.9 \%, 22.6 \%, 20.0 \%$ and $8.0 \%$ in years 1 to 5 , respectively.

IOP was significantly lower at all examined post-operative time points $(41.1 \%, 39.3 \%, 43.3 \%, 46.4 \%$ and $45.3 \%$ at years 1 to 5 , respectively, $p<0.001$ at all time points).

Four eyes (5.7\%) failed to meet any of the success criteria. Of these, 3 eyes $(4.3 \%)$ required further glaucoma surgery and one eye (1.4\%) progressed to no perception of light at 48 months. No patients had an IOP $\leq 5 \mathrm{mmHg}$ on two consecutive occasions after 3 months.

Conclusion Viscocanalostomy and phaco-viscocanalostomy are a safe and effective surgical option in the management of chronic narrow angle glaucoma.
\end{abstract}

Keywords Glaucoma $\cdot$ Narrow angle $\cdot$ Viscocanalostomy $\cdot$ Phaco-viscocanalostomy $\cdot$ Non-penetrating glaucoma surgery

Tasmin Berman

Tasminberman@nhs.net

1 St Pauls Eye Unit, Liverpool University Hospital, Liverpool, UK 


\section{Key messages}

- Management of chronic narrow angle glaucoma had traditionally involved cataract surgery alone, trabeculectomy with Mitomyocin $\mathrm{C}$ or a combined procedure. Viscocanalostomy has lower complication rates compared to trabeculectomy and can be combined with cataract surgery without affecting clinical outcome.

- Viscocanalostomy achieves a significant and sustained reduction in intraocular pressure and drop usage at 5 years post-operatively in narrow angle glaucoma.

- Patients undergoing viscocanalostomy for narrow angle glaucoma require less post-operative intervention and fewer post-operative clinic visits, which in the current climate with COVID 19 helps reduce the risk to patients and staff as well as reducing the strain on the health care system.

\section{Introduction}

Glaucoma is the most common cause of preventable vision loss and accounted for almost one-third of the new vision impairment certificates in England from 2017 to 2018 [1]. Primary open-angle glaucoma is three times more common than chronic angle-closure glaucoma, although angle closure is responsible for $50 \%$ of blindness caused by glaucoma. The primary risk factors for angle closure include older age, female gender and Asian or Eskimoan race [2]. The global prevalence of primary angle closure was reported as $0.6 \%$ in a systemic review by Zhang et al. [3].

Management of chronic narrow angle glaucoma has traditionally involved cataract surgery alone [4, 5], trabeculectomy with mitomycin C (MMC) [6] or a combined procedure [7-9]. Cataract surgery alone might not be as effective in satisfactorily reducing the intraocular pressure (IOP) when patients are poorly controlled with topical medication [7, 8]. Combined surgery has also been shown to be more cost-effective than cataract surgery alone [7, 9].

Glaucoma services within the hospital eye service were stretched to breaking point even before the COVID19 pandemic with up to 22 patients per month suffering permanent and severe vision loss due to delayed followup $[10,11]$. We are now having to change our current practice to try and reduce the risk to patients and staff as well as reduce the strain on the healthcare system as a whole [12].

Compared to trabeculectomy, non-penetrating glaucoma surgery (NPGS) has been shown to have a lower incidence of complications with comparable long-term success rates [13-16]. In addition, due to the reduced need for additional interventions and complications post-operatively, patients may require less follow-up appointments [17] which might help reduce the strain on the already overwhelmed system. However, there is limited literature for NPGS in narrow angle glaucoma. There have been no previous studies except for a small series by Yuen et al. of 29 eyes undergoing combined phacoemulsification and non-penetrating deep sclerectomy [18].

In this study, we assessed the outcomes of viscocanalostomy (VC) and phaco-viscocanalostomy (Phaco-VC) in patients with narrow angle glaucoma to evaluate success in terms of IOP and the post-operative interventions undertaken to achieve this.

\section{Method}

All patients with narrow angle glaucoma undergoing VC and Phaco-VC between 2010 and 2017 under a single surgical team at the Royal Liverpool University Hospital were included from a prospective database.

The technique involved a superior corneal traction suture with 7-0 vicryl. A fornix-based conjunctival flap was raised superiorly, and haemostasis was achieved. A two-third depth scleral thickness limbal-based flap measuring $5 \times 5 \mathrm{~mm}$ was raised and advanced into clear cornea. A deep $4 \times 4 \mathrm{~mm}$ scleral flap was dissected to the level of Schlemm's canal, deroofing it and extended into the corneal stroma at the level of Descemet's membrane to create the Trabeculo-Descemet window (TDW). Viscoat ${ }^{\circledR}$ (sodium hyaluronate and sodium chondroitin sulphate) was injected onto the canal and applied to the scleral bed. The deep flap was excised close to the TDW. The superficial scleral flap and conjunctiva were closed tightly with $10-0$ vicryl sutures. Where there were peripheral anterior synechiae (PAS) blocking the TDW, an intentional peripheral iridectomy (PI) was performed. No antimetabolite was used. 
Patients were excluded if they did not have 12 months of follow-up. There were no other exclusion criteria. An intervention was defined as any procedure carried out after the VC to assist with the success of the surgery. In this series this was Nd:YAG laser goniopuncture (Yag GP).

The primary outcome was a change in IOP. This was analysed from a prospectively maintained surgical outcome database. Secondary outcomes were post-operative complications and interventions and the number of glaucoma drops used. Change in visual acuity (VA) and visual field (VF) mean deviation (MD) were also noted.

Subgroup analysis was performed to look at outcomes when VC was combined with cataract surgery.

SPSS v22 (SPSS, Chicago, IL) was used to perform statistical analysis. Normality of data (IOP, drops, visual acuity (VA) and mean deviation (MD)) was examined using the Kolmogorov-Smirnov test. A linear mixed model with Bonferroni correction was used to examine for differences in IOP and drops between pre-operative and post-operative years 1-5. Change in VA and visual field MD pre- and post-operatively were examined using the Wilcoxon signed rank test. The study conduct adhered to the tenets of the Declaration of Helsinki. No formal ethical approval was required because it was a retrospective study.

\section{Success definition}

Success was defined at two IOP cut-off points: IOP $\leq 21 \mathrm{mmHg}$ and IOP $\leq 15 \mathrm{mmHg}$. Complete surgical success was defined as IOP $\leq 21$ or $\leq 15 \mathrm{mmHg}$ with no additional medications and qualified surgical success as IOP $\leq 21$ or $\leq 15 \mathrm{mmHg}$ with additional glaucoma medications. Failure was defined as IOP $>21 \mathrm{mmHg}$ on two consecutive visits, IOP $\leq 5 \mathrm{mmHg}$ on two consecutive occasions after 3 months (numerical hypotony as described by the World Glaucoma Association's Guidelines on Design and Reporting Glaucoma Surgical Trials [19]), any repeat operation for glaucoma or loss of light perception.

\section{Results}

Eighty-five eyes of 54 patients were included from the database. Fifteen eyes ( 8 patients) were excluded due to lack of 12 months of data. Of these patients, 3 patients died, 2 patients were followed up in their local hospital, 2 patients failed to attend follow-up multiple times and 1 patient was followed up in the private sector. Therefore,

Table 1 Patient demographics

\begin{tabular}{ll}
\hline & Mean \pm SD (range) \\
\hline Age (years) & $71.9 \pm 10.5(47-92)$ \\
Male:female (\%) & $37: 63$ \\
Follow-up (months) & $41.31 \pm 17.91(12-60)$ \\
Pre-operative IOP (mmHg) & $25.73 \pm 9.59(14-58)$ \\
Pre-operative drops (number) & $3.03 \pm 1.12(0-5)$ \\
Pre-operative visual acuity (Log MAR) & $0.23 \pm 0.43(-0.1-2.2)$ \\
Pre-operative mean deviation (dB) & $-8.76 \pm 8.03(1.6$ to -26.79$)$ \\
Procedure performed & \\
Viscocanalostomy & $24(34 \%)$ \\
Phaco-viscocanalostomy & $46(66 \%)$ \\
Previous procedures & \\
None & $4(5.7 \%)$ \\
Peripheral iridotomy & $64(91.4 \%)$ \\
Phacoemulsification & $8(11.4 \%)$ \\
Combined phacoemulsification, vitrectomy and anterior hyaloidectomy & $2(2.9 \%)$ \\
Argon laser peripheral iridotomy & $2(2.9 \%)$ \\
Cyclodiode & $1(1.4 \%)$ \\
Pan retinal photocoagulation for diabetic retinopathy & $2(2.9 \%)$ \\
Glaucoma type & \\
Chronic narrow angle glaucoma & $59(84.3 \%)$ \\
Acute angle-closure glaucoma & $5(7.1 \%)$ \\
Ocular hypertension with narrow angles & $3(4.3 \%)$ \\
Normal tension glaucoma with narrow angles & $1(1.4 \%)$ \\
\hline & $2(2.9 \%)$ \\
\hline
\end{tabular}


Fig. 1 A graph showing mean intraocular pressure (IOP) and mean number of drops preoperatively (Pre-op) and at all post-operative time points

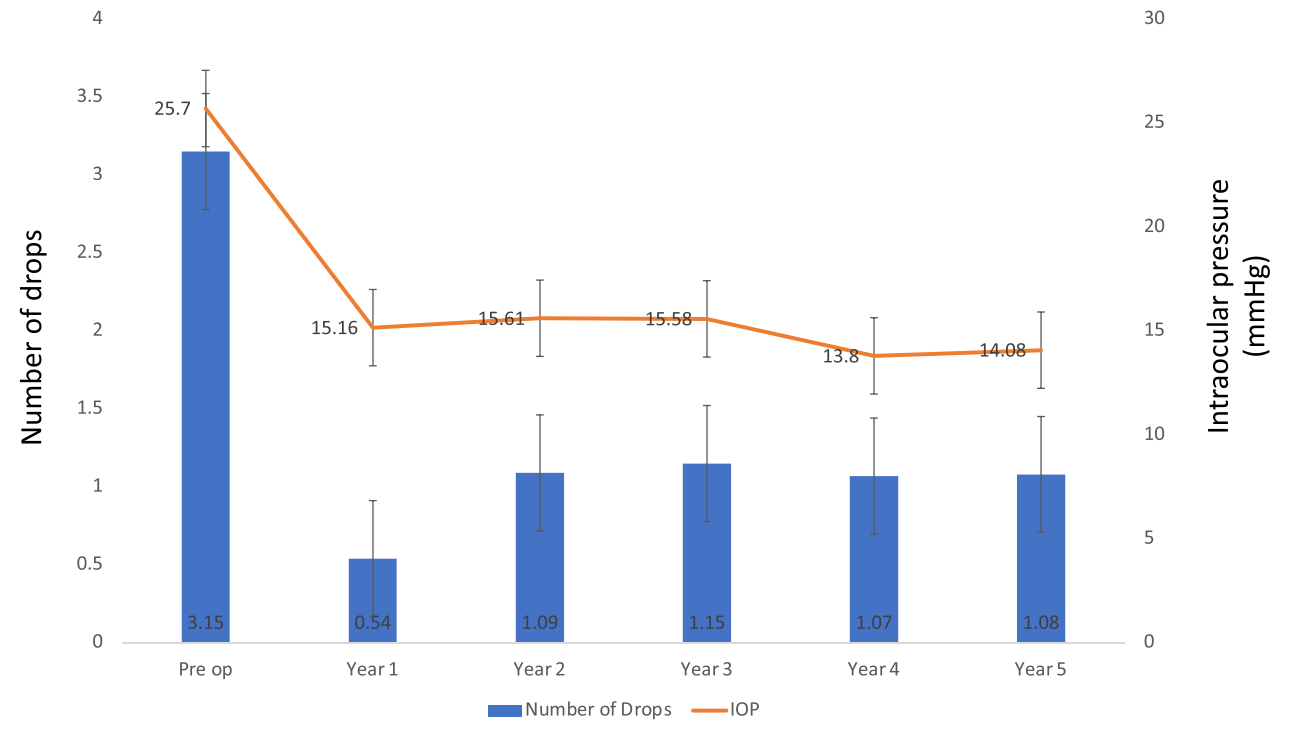

Table 2 Descriptive statistics of mean intraocular pressure (IOP) and drops pre-operatively and at all post-operative time points including number of patients per year

\begin{tabular}{lllllll}
\hline & Pre-op (70) & Year 1 (69) & Year 2 (67) & Year 3(53) & Year 4 (45) & Year 5 (25) \\
\hline IOP (mmHg) & $25.73 \pm 9.7$ & $15.16 \pm 3.59$ & $15.46 \pm 3.76$ & $14.58 \pm 3.46$ & $13.80 \pm 3.17$ & $14.08 \pm 2.61$ \\
Drops (number) & $3.15 \pm 1.14$ & $0.54 \pm 0.93$ & $1.03 \pm 1.11$ & $1.15 \pm 1.02$ & $1.07 \pm 0.96$ & $1.08 \pm 1.00$ \\
\hline
\end{tabular}

Table 3 Complete and qualified success for intraocular pressure $\leq 15 \mathrm{mmHg}$ and intraocular pressure $\leq 21 \mathrm{mmHg}$ (number of patients and percentage) at each time point post-operatively

Fig. 2 Kaplan-Meier survival plot for complete (defined as intraocular pressure $\leq 21 \mathrm{mmHg}$ with no medication) and qualified (defined as intraocular pressure $\leq 21 \mathrm{mmHg}$ with additional glaucoma medications) success

\begin{tabular}{llccccc}
\hline & IOP & Year 1 & Year 2 & Year 3 & Year 4 & Year 5 \\
\hline Qualified success & $\leq 21 \mathrm{mmHg}$ & $65 / 69$ & $59 / 67$ & $49 / 53$ & $41 / 45$ & $23 / 25$ \\
(with additional & & $94.2 \%$ & $88.1 \%$ & $92.5 \%$ & $91.1 \%$ & $92.0 \%$ \\
medication) & $\leq 15 \mathrm{mmHg}$ & $37 / 69$ & $42 / 67$ & $37 / 53$ & $31 / 45$ & $15 / 25$ \\
& & $53.6 \%$ & $60.9 \%$ & $69.8 \%$ & $68.9 \%$ & $64.0 \%$ \\
Complete success & $\leq 21 \mathrm{mmHg}$ & $44 / 69$ & $25 / 67$ & $16 / 53$ & $10 / 45$ & $6 / 25$ \\
(with no medica- & & $63.8 \%$ & $37.3 \%$ & $30.2 \%$ & $22.2 \%$ & $24.0 \%$ \\
tion) & $\leq 15 \mathrm{mmHg}$ & $27 / 69$ & $18 / 67$ & $12 / 53$ & $9 / 45$ & $2 / 25$ \\
& & $39.1 \%$ & $26.9 \%$ & $22.6 \%$ & $20.0 \%$ & $8.0 \%$ \\
\hline
\end{tabular}

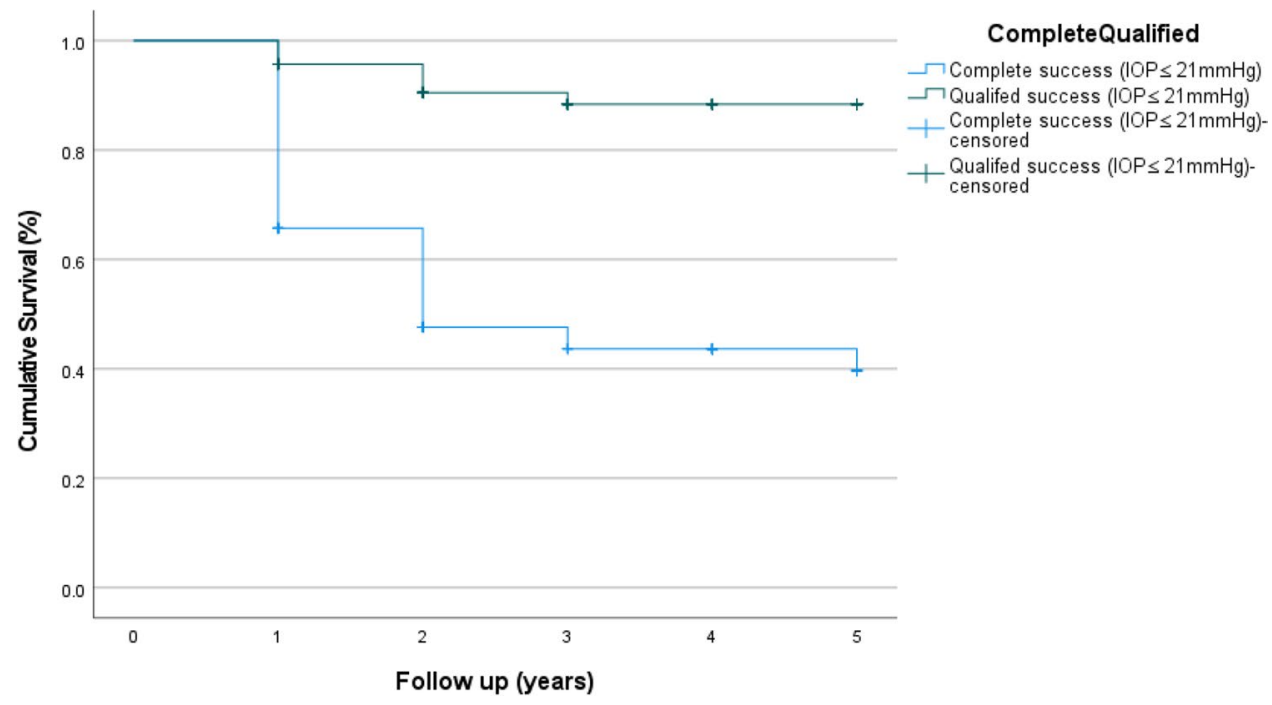


70 eyes of 46 patients were included in the final analysis. Patient demographics are shown in Table 1. Forty-six eyes had Phaco-VC and 24 eyes had VC alone (Table 1); 10 eyes were pseudophakic pre-operatively in the latter group.

\section{IOP outcome}

IOP was significantly lower at all examined post-operative time points (year 1 by $41.1 \%$, year 2 by $39.3 \%$, year $343.3 \%$, year $446.4 \%$ and year $545.3 \%, p<0.001$ at all time points) compared to pre-operative values. The number of post-operative glaucoma drops was also significantly reduced compared to that pre-operatively at all time points $(p<0.001)$ (Fig. 1 and Table 2). Of the patients that required post-operative glaucoma drops, the majority were on mono or dual therapy $(77.2 \%, 74.3 \%, 80.5 \%, 83.8 \%$ and $77.8 \%$ for years 1 to 5 respectively). One patient was lost to follow-up at 1 year and reappeared at 2 years.

Qualified success for an IOP $\leq 21 \mathrm{mmHg}$ was achieved in $94.2 \%, 88.1 \%, 92.5 \%, 91.1 \%$ and $92.0 \%$ and complete success in $63.8 \%, 37.3 \%, 30.2 \%, 22.2 \%$ and $24.0 \%$ in years 1 to 5, respectively. Qualified success for an IOP $\leq 15 \mathrm{mmHg}$ was achieved in $53.6 \%, 60.9 \%, 69.8 \%, 68.9 \%$ and $64.0 \%$ and complete success in $39.1 \%, 26.9 \%, 22.6 \%, 20.0 \%$ and $8.0 \%$ in years 1 to 5 , respectively (Table 3 ).

Kaplan-Meier survival curves were significantly different for complete and qualified success with IOP $\leq 21 \mathrm{mmHg}$ $(p=0.001$, Fig. 2$)$ but there was no significant difference between the outcomes of patient who had VC alone and those that had a Phaco-VC $(p=0.181)$.

Table 4 Intra- and post-operative complications

\begin{tabular}{ll}
\hline Complication type & Number of patients \\
\hline Trabeculo-Descemet's window perforation & $12 / 70(17.1 \%)$ \\
$\begin{array}{l}\text { Scleral flap perforation during surgery repaired } \\
\quad \text { with Tutoplast }\end{array}$ & $2 / 70(2.9 \%)$ \\
$\begin{array}{l}\text { Corneal perforation with traction suture } \\
\text { Bandage contact lens post-operatively due to }\end{array}$ & $1 / 70(1.4 \%)$ \\
$\quad$ shallow anterior chamber & $10 / 70(14.3 \%)$ \\
\hline
\end{tabular}

\section{Visual acuity outcome}

VA was stable for the vast majority of patients (59 eyes, $84.3 \%$ ). This was defined as a change of less than $0.2 \mathrm{Log}-$ MAR. There was no significant difference between pre- and post-operative VA $(p=0.831)$.

Eleven eyes lost more than 0.2 LogMAR. This was due to glaucoma in 7 patients, decompensated cornea in 1, proliferative diabetic retinopathy in 1 , epiretinal membrane in 1 and retinal vein occlusion with cystoid macular oedema in 1 .

\section{Visual fields}

Thirteen eyes did not have a visual field test recorded; this was either because they were unable to perform them reliably, or they were not available for review in the patients' record. Pre-operatively, of the 57 eyes that had a visual field performed, 21 eyes had advanced visual field loss pre-surgery, 5 had moderate loss and 31 had mild loss (mild $\geq-6 \mathrm{~dB}$; moderate -6 to $-12 \mathrm{~dB}$ and advanced $\leq-12 \mathrm{~dB}$ ). Post-operatively, 48 eyes had visual fields available at final follow-up. Mean post-operative $\mathrm{MD}$ was $-9.79 \mathrm{~dB} \pm 9.03 \mathrm{~dB}$ compared to $-8.76 \pm 8.03$ pre-operatively. There was no statistical difference between pre- and post-operative MD $(p=0.415)$.

\section{Cataract surgery}

Twenty-four patients underwent VC, of which 10 (41.7\%) were pseudophakic at the time of surgery. Four (28.6\%) out of 14 eyes that were phakic at the time of their VC underwent cataract surgery by the end of the study period.

\section{Intentional peripheral iridectomy}

Sixty-six eyes had already had a pre-operative laser peripheral iridotomy. Fourteen eyes (20\%) had an intentional PI at the time of surgery due to PAS at the TDW; this included all patients that had not had a pre-operative laser peripheral iridotomy.

Table 5 Patients that progressed to failure

\begin{tabular}{llllllllll}
\hline $\begin{array}{l}\text { Type of } \\
\text { glaucoma }\end{array}$ & Pre-op IOP & $\begin{array}{l}\text { Pre-op MD } \\
(\mathrm{dB})\end{array}$ & Pre-op drops & $\begin{array}{l}\text { Pre-op } \\
\text { BCVA }\end{array}$ & $\begin{array}{l}\text { BCVA final } \\
\text { FU }\end{array}$ & $\begin{array}{l}\text { Failure } \\
\text { (months) }\end{array}$ & $\begin{array}{l}\text { IOP at point } \\
\text { time of } \\
\text { failure }\end{array}$ & IOP final FU Procedure \\
\hline CNAG & 28 & -3.98 & 4 & $6 / 6$ & $6 / 9$ & 8 & 22 & 14 & Ahmed valve \\
CNAG & 53 & -24.3 & 4 & $6 / 18$ & $6 / 36$ & 23 & 31 & 18 & Ahmed valve \\
CNAG & 22 & -8.92 & None & $6 / 5$ & $6 / 36^{*}$ & 28 & 21 & 15 & Repeat VC \\
CNAG & 20 & -26.97 & 4 & HM & NPL & 48 & 16 & 11 & None \\
\hline
\end{tabular}

$C N A G$, chronic narrow angle glaucoma; $I O P$, intraocular pressure; $F U$, follow-up; * from cataract and central retinal vein occlusion; $V C$, viscocanalostomy 


\section{Complications}

Table 4 shows intraoperative and post-operative complications. No patients had any long-term complications.

\section{Post-operative intervention}

Seven patients (10.8\%) required Yag GP following surgery, which was performed between 6 and 36 months.

\section{Failure}

Four eyes (5.7\%) failed to meet any of the above success criteria (Table 5). Of these, 3 eyes (4.3\%) required further glaucoma surgery due to poorly controlled IOP; one at 8 months (Ahmed valve), one at 23 months (Ahmed valve) and one at 28 months (repeat VC). One patient (1.4\%) progressed to no perception of light (NPL) at 48 months. This patient had a best corrected visual acuity (BCVA) of hand movements (HM) at time of listing and end stage glaucoma. No patients had an IOP $\leq 5 \mathrm{mmHg}$ on two consecutive occasions after 3 months.

\section{Discussion}

We report our results of un-augmented VC and Phaco-VC in patients with narrow angle glaucoma. $\mathrm{VC}$ was able to achieve qualified success with an IOP $\leq 21 \mathrm{mmHg}$ in $92 \%$ of patients at 5 years with a $45.3 \%$ reduction in IOP from baseline. The number of glaucoma drops was also significantly reduced compared to that pre-operatively at all time points. To our knowledge, our study provides the longest follow-up for the largest number of eyes undergoing $\mathrm{VC}$ for narrow angle glaucoma.

There is limited evidence for the outcome of NPGS for narrow angle glaucoma. In 2007, Yuen et al. [18] reported their outcomes for 29 eyes undergoing deep sclerectomy combined with phacoemulsification in patients with chronic angle-closure glaucoma. The authors reported that $86 \%$ of eyes had a qualified success with IOP $\leq 21 \mathrm{mmHg}$ and a reduction in IOP from $20.3 \pm 3.9 \mathrm{mmHg}($ mean $\pm \mathrm{SD}$ ) preoperatively to $15.9 \pm 3.1 \mathrm{mmHg}$ post-operatively at the last follow-up visit $(p<0.001)$. In our cohort, IOP reduction was maintained significantly below pre-operative levels up to 5 years after surgery.

We have previously reported our results of qualified (87.5-90.2\%) and complete success (78-90\%) rates in a cohort of patients with POAG [14] and qualified (80-95\%) and complete success (30.6-52.5\%) for a cohort of patients with advanced glaucoma [16] undergoing VC and Phaco-VC.

Trabeculectomy is still considered the gold standard surgical treatment for glaucoma and has better IOP control than
VC [20]. NPGS however has significant benefits both for the patient and the operating team with reduced need for post-operative interventions, lower rates of sight-threatening complications and a better quality of life for patients [20]. A multicentre analysis of trabeculectomies by Kirwan et al. [21] suggested that the initial trabeculectomy was just the beginning of the patient's surgical journey and that the majority of patients required frequent post-operative interventions.

There are no direct comparative trials comparing NPGS and trabeculectomies in narrow angle glaucoma. Romero et al. [22] reported the outcomes of trabeculectomy for primary angle-closure glaucoma. Qualified success rates $( \pm \mathrm{SD})$ after 1,3 and 5 years of follow-up for IOP $<18 \mathrm{mmHg}$ were $92 \%( \pm 2.2 \%), 78 \%( \pm 3.8 \%)$ and $72 \%( \pm 4.3 \%)$, respectively and for IOP $<15 \mathrm{mmHg}, 86 \%( \pm 3.0 \%), 65 \%( \pm 4.4 \%)$ and $59 \%( \pm 4.7 \%)$, respectively which are comparable to our results. Although our IOP reduction was not as low, our pre-operative IOP was higher $(25.73 \pm 9.7)$ compared to $18.8( \pm 3.5)$ in the study by Romero et al. [22]. In addition, $16.9 \%$ of patients from this study required additional surgery (second trabeculectomy (47.8), glaucoma drainage device (39.1\%) and bleb revision (13\%)) compared with $4.3 \%$ of our patients requiring further glaucoma surgery and $10.8 \%$ requiring a Yag GP. Song et al. [23] reported 12-month data for trabeculectomy and combined phaco-trabeculectomy in primary angle-closure glaucoma and the qualified success rate 12 months after surgery was $65.7 \% \pm 7.2 \%$, for IOP $<21 \mathrm{mmHg}$ and $38.2 \% \pm 7.4 \%$, for IOP $<15 \mathrm{mmHg}$. Our 1-year data is more favourable than this. Our complete success rates were lower than the results for trabeculectomy in the literature. However, our qualified success rates are comparable, and the majority of patients were controlled on dual (76\%) or mono (52\%) therapy post-operatively.

Previous studies suggest a collapse of the Schlemm's canal with raised IOP [24]. There is expansion of the trabecular meshwork into the Schlemm's canal (SC) with raised IOP which is reversed with VC [25]. Hong et al. [26] also suggested a similar mechanism for trabeculectomies so the mechanism by which VC helps in narrow angle glaucoma might be through SC dilatation.

Cataract formation is also a well reported complication after trabeculectomy. It has been reported that trabeculectomies increase the risk of cataract formation by $78 \%$ [27]. In our study, of those patients who had a VC and were phakic, 4 patients $(28.6 \%)$ went on to require a cataract surgery during the study period. However, this is a small cohort (14 eyes in total) to make assumptions from. Cataract surgery has been shown to increase the need for post-operative interventions and glaucoma drops reducing the success of trabeculectomies in $30-39 \%$ of cases $[28,29]$. There was no difference in success rates in our study between VC and PhacoVC. Although our numbers for VC were small, a similar 
conclusion was made by Wishart et al. [14]. In addition, combined surgery has also been shown to be more costeffective [9] than cataract surgery alone in controlling IOP in narrow angle glaucoma patients.

NPGS have a greater safety profile with a lower risk of complications compared to trabeculectomy [30-33]. A Cochrane review reported fewer complications with NPGS (17\%) compared to trabeculectomy (65\%) [20]. The UK national trabeculectomy surgery survey [34] reported early complications in $46.6 \%$ and late complications in $42.3 \%$ of cases. Trabeculectomy in narrow angles is particularly challenging, further increasing the risk of complications $[7,8]$. In our study, 10 patients (14.3\%) had early post-operative complications, with a shallow anterior chamber requiring a bandage contact lens. These had resolved in all patients by 2 weeks post-operatively. There were no long-term complications. This is significantly lower than the results for trabeculectomy with $2.6 \%$ requiring resuturing or revision for significant early hypotony and a $0.5-3.9 \%$ incidence of blebitis and $0.5 \%$ incidence of bleb-related endophthalmitis (Kirwan et al. [21], Tham et al. [7]).

There was no statistical difference between pre- and postoperative visual field MD $(p=0.145)$. In addition, we found no difference between success and severity of glaucoma based on visual field MD. Not all patients had visual fields performed for a variety of reasons and the number of patients completing visual fields reduced year on year. This may be expected with time [35]; however, $68 \%$ of patients were able to complete visual fields at their final follow-up. This is higher than in our previously reported paper [16] where only $45.2 \%$ of patients had a visual field completed at their final follow-up. This is likely because $44.29 \%$ of our cohort had mild glaucoma based on visual field MD and therefore will be likely to be able to continue performing visual fields for longer compared to advanced glaucoma.

Seven patients $(10.8 \%)$ required a post-operative intervention of Yag GP. This was performed between 6 and 36 months. This is in line with other studies in the literature $[16,18]$.

Of the 4 eyes that failed, 2 of the eyes had advanced glaucoma pre-operatively and one of these with a vision of hand movements at presentation and progressed to no perception of light. One other patient was lost to follow-up and reappeared with progression requiring surgery. The final patient had further surgery for sub-optimal IOP with good visual acuity and IOP outcome. These results are inkeeping with the results from our advanced glaucoma and viscocanalostomy study [16] and better than the results reported by Yuen et al. [18].

Limitations of this study include data being retrospectively analysed and there being no control group. However, data was collected from a prospectively maintained database and a significant proportion of patients were followed up to 4 years. In addition, we have included data from both eyes of patients where available in our analysis, this can potentially affect the statistical analysis especially as there is not equal numbers of patients having both eyes operated on in the Phaco and Phaco-VC groups. However, we are not comparing risk factors between the two groups so feel this would not have a significant effect on the final data. It would also have been desirable to have a higher number of patients completing a visual field test both pre- and postoperatively to further strengthen the study.

To our knowledge, this is the longest study reporting the effectiveness of un-augmented VC in narrow angle glaucoma to date. $\mathrm{VC}$ was able to achieve an IOP $\leq 21$ in up to $92 \%$ patients for a period of up to 5 years. VC has similar qualified success rates as trabeculectomy with MMC with a good safety profile and minimal requirement for postoperative interventions. This is particularly important in the current climate with COVID-19 where we want to keep attendances to a hospital setting to a minimum $[11,12,17]$.

Author contribution All authors contributed to the study conception and design. Material preparation, data collection and analysis were performed by Tasmin Berman and Tobi Somerville. The first draft of the manuscript was written by Tasmin Berman, and all authors commented on previous versions of the manuscript. All authors read and approved the final manuscript.

Code availability Non-applicable.

\section{Declarations}

Ethics approval This is a retrospective study. For this type of study, formal consent is not required and all the procedures performed were part of the routine care. Therefore, ethical approval is not required.

Conflict of interest The authors declare no competing interests.

\section{References}

1. Rahman F, Zekite A, Bunce C et al (2020) Recent trends in vision impairment certifications in England and Wales. Eye 34:1271-1278

2. European Glaucoma Society Terminology and Guidelines for Glaucoma, 4th Edition (2017) Chapter 2: classification and terminology supported by the EGS Foundation: part 1: foreword; introduction; glossary; chapter 2 classification and terminology. Br J Ophthalmol. 101(5):73-127

3. Zhang N, Wang J, Chen B et al (2021) Prevalence of primary angle closure glaucoma in the last 20 years: a meta-analysis and systemic review. Front Med 7:1006

4. Gunning FP, Greve EL (1998) Lens extraction for uncontrolled angle-closure glaucoma: long-term follow-up. J Cataract Refract Surg 24:1347-1356

5. Lai JS, Tham CC, Chan JC (2006) The clinical outcomes of cataract extraction by phacoemulsification in eyes with primary 
angle-closure glaucoma (PACG) and co-existing cataract: a prospective case series. J Glaucoma 15:47-52

6. Rodriquez-Una I, Azuara-Blanco A, King AJ (2017) Survey of glaucoma surgical preferences and post-operative care in the United Kingdom. Clin Exp Ophthalmol 45:232-240

7. Tham C, Kwong Y, Leung D et al (2009) Phacoemulsification verses combined phacotrabeculectomy in medically uncontrolled chronic angle closure glaucoma with cataracts. American Academy of Ophthalmology 116:725-731

8. Hansapinyo L, Choy B, Lai J et al (2020) Phacoemulsification verses phacotrabeculectomy in primary angle closure glaucoma with cataract: long-term clinical outcomes. J Glaucoma 29:15-23

9. Chan PP, Li EY, Tsoi KKF et al (2017) Cost-effectiveness of phacoemulsification verses combined phacotrabeculectomy for treating primary angle closure glaucoma. J Glaucoma 26:911-922

10. Foot B, MacEwen C (2017) Surveillance of sight loss due to delay in ophthalmic treatment or review: frequency, cause, and outcome. Eye 31:771-775

11. Jayaram H, Strouthidis NG, Gazzard G (2020) The COVID-19 pandemic will redefine the future delivery of glaucoma care. Eye 34:1203-1205

12. Husain R, Zhang X, Aung T (2020) Challenges and lessons for managing glaucoma during COVID-19 pandemic: perspectives from Asia. Ophthalmology 9:e63-e64

13. Choudhary A, Wishart PK (2007) Non-penetrating glaucoma surgery augmented with mitomycin $\mathrm{C}$ or 5 -fluorouracil in eyes at high risk of failure of filtration surgery: long-term results. Clin Exp Ophthalmol 35:340-347

14. Wishart PK, Wishart MS, Choudhary A et al (2008) Long-term results of viscocanalostomy in pseudoexfoliative and primary open angle glaucoma. Clin Exp Ophthalmol 36:148-155

15. Shaarawy T, Nguyen C, Schnyder C et al (2003) Five-year results of viscocanalostomy. Br J Ophthalmol 87:441-445

16. Tsagkataki M, Bampouras TM, Choudhary A (2018) Outcomes of viscocanalostomy and phaco-viscocanalostomy in patients with advanced glaucoma. Clin Exp Ophthalmol 256:1482-1487

17. Dwivedi R, Somervile T, Cheeseman R et al (2021) Deep sclerectomy and trabeculectomy augmented with mitomycin C: 2-year post-operative outcomes. Clin Exp Ophthalmol 259:1965-1974

18. Yuen N, Chan O, Hui S et al (2007) Combined phacoemulsification and non-penetrating deep sclerectomy in the treatment of chronic angle-closure glaucoma with cataract. Eur J Ophthalmol $17: 208-215$

19. Jampel HD (2009) Reporting post-operative complications in glaucoma surgical trials In: Shaarawy TMS, Sherwood MB, Grehn F. (Eds.), Guidelines on design and reporting of glaucoma surgical trials, Kugler Publications, Amsterdam, the Netherlands pp. 33-39

20. Eldaly MA, Bunce C, Elsheikha OZ, etal.,(2014) Non-penetrating filtration surgery versus trabeculectomy for open-angle glaucoma. Cochrane Database Syst Rev2
21. Kirwan JF, Lockwood AJ, Shah P et al (2013) Trabeculectomy outcomes group audit study group trabeculectomy in the 21 st century: a multicentre analysis. Ophthalmology 120:2532-2539

22. Romero P, Hirunpatravong P, Alizadeh R et al (2018) Trabeculectomy with mitomycin-C: outcomes and risk factors for failure in primary angle-closure glaucoma. J Glaucoma 27:101-107

23. Song BJ, Ramanathan M, Morales E et al (2016) Trabeculectomy and combined phacoemulsification-trabeculectomy: outcomes and risk factors for failure in primary angle closure glaucoma. J Glaucoma 25:763-769

24. Kagemann L, Wang B, Wollstein G et al (2014) IOP elevation reduces Schlemm's canal cross-sectional area. Invest Ophthalmol Vis Sci 55(3):1805-1809

25. Carreon T, Van der Merwe E, Fellmen R et al (2017) Aqueous outflow - a continuum from trabecular meshwork to episcleral veins. Prog Retin Eye Res 57:108-133

26. Hong J, Yang Y, Wei A, etal.,(2014) Schlemm's canal expands after trabeculectomy in patients with primary angle-closure glaucoma. Invest Ophthalmol Vis Sci. 12;55(9):5637-42.

27. AGIS (Advanced Glaucoma Intervention Study) Investigators (2001) The advanced glaucoma intervention study: 8 risk of cataract formation after trabeculectomy. Arch Ophthalmol 119:1771-1779

28. Husain R, Liang S, Foster PJ et al (2012) Cataract surgery after trabeculectomy: the effect on trabeculectomy function. Arch Ophthalmol 130:165-170

29. Longo A, Uva MG, Reibaldi A et al (2015) Long-term effect of phacoemulsification on trabeculectomy function. Eye 29:1347-1352

30. Ang GS, Varga Z, Shaarawy T (2010) Postoperative infection in penetrating versus non-penetrating glaucoma surgery. Br J Ophthalmol 94:1571-1576

31. Mendrinos E, Mermoud A, Shaarawy T (2008) Non-penetrating glaucoma surgery. Surv Ophthalmol 53:592-630

32. Ahmed II, Shaarawy T (2004) Viscocanalostomy versus trabeculectomy. Ophthalmology 111:1066-1067

33. Tan JC, Hitchings RA (2001) Non-penetrating glaucoma surgery: the state of play. Br J Ophthalmol 85:234-237

34. Edmunds B, Thompson JR, Salmon JF et al (2002) The national survey of trabeculectomy III early and late complications. Eye 16:297-303

35. Saunders LJ, Russell RA, Kirwan JF (2014) Examining visual field loss in patients in glaucoma clinics during their predicted remaining lifetime. Invest Ophthalmol Vis Sci 255:102-109

Publisher's note Springer Nature remains neutral with regard to jurisdictional claims in published maps and institutional affiliations. 